\title{
Investigation on the Adsorption and Photooxidation of Glycerol at $\mathrm{TiO}_{2}$ Nanotubular Arrays
}

\author{
Simonetta Palmas, ${ }^{1}$ Anna Da Pozzo, ${ }^{1}$ Michele Mascia, ${ }^{1}$ \\ Annalisa Vacca, ${ }^{1}$ and Roberto Matarrese $^{2}$ \\ ${ }^{1}$ Dipartimento di Ingegneria Meccanica, Chimica e Materiali, Università degli Studi di Cagliari, Piazza d'Armi, 09123 Cagliari, Italy \\ ${ }^{2}$ Dipartimento di Energia, Politecnico di Milano, Piazza L. Da Vinci 32, 20133 Milano, Italy
}

Correspondence should be addressed to Simonetta Palmas, simonetta.palmas@dimcm.unica.it

Received 1 June 2012; Accepted 18 July 2012

Academic Editor: Manickavachagam Muruganandham

Copyright (c) 2012 Simonetta Palmas et al. This is an open access article distributed under the Creative Commons Attribution License, which permits unrestricted use, distribution, and reproduction in any medium, provided the original work is properly cited.

\begin{abstract}
A study is presented on the adsorption of glycerol at $\mathrm{TiO}_{2}$ as well as on its oxidative process during the contemporary water Photoelectro-splitting for hydrogen production. A deepening in the understanding on the working mechanism of the $\mathrm{TiO}_{2}$ nanotubular photoanodes and on the interactions between glycerol and these structures has been gained through photocurrent tests, voltammetric scans, and EIS analysis. A range of wavelength of the incident radiation is investigated from 340 to $400 \mathrm{~nm}$ at which the effect of glycerol on the photocurrent is measured. Quantitative analysis of the EIS results is performed by the equivalent circuit approach.
\end{abstract}

\section{Introduction}

The growing attention on innovative processes using glycerol as starting material for the obtainment of more valuable products such as other chemicals or energy is due to the great increase of its market availability as byproduct in the biodiesel production [1]. Since the international legislation is addressed to a greater production of bio- or renewable fuels to be used for transportation, the crude glycerol production from the biodiesel industry is increasing exponentially. Consequently, the commercial price of glycerol is greatly lowered and several perspectives of research for its use are of big interest [2-5]. To face this issue, following the principles of sustainable chemistry means to pay attention to avoid the use and the generation of harmful substances, to reduce the energy consumption, and to use renewable resources such as solar energy.

In this context, a very interesting way to use this chemical is in the photo-electrocatalytic hydrogen production, conceiving a system which can couple, under a suitable combination between irradiation and external potential bias, the cathodic hydrogen production and the oxidation of glycerol, used as sacrificial reagent at the photoanode. Actually, among the possible ways to increase the efficiency of a photoelectrochemical cell, the presence of a sacrificial agent into the solution must be considered as effective to prevent the electronhole recombination process.

For photocatalytic hydrogen generation, compounds such as alcohols, EDTA, or ions such as $\mathrm{I}^{-}, \mathrm{IO}_{3}{ }^{-} \mathrm{CN}^{-}$, and $\mathrm{Fe}^{3+}$, but also many other organic pollutants, such as oxalic acid, formic acid, and formaldehyde, can be used as sacrificial reagents ([6-8] and references therein).

As above reported, due to its wide market availability, glycerol has been proposed in the present paper as sacrificial agent; moreover, a further enhancement of the process could consider the possibility to make the oxidation of glycerol selective in order to address the process to obtain its more valuable oxidation byproducts such as dihydroxyacetone, glyceric acid, or glyceraldehyde.

In the field of photo-electrocatalytic hydrogen production, the use of photoanodes based on nanotubular $\mathrm{TiO}_{2}$ has been assessed by several studies that highlighted their optimal properties in terms of corrosion resistance, reactivity, photoactivity, low cost, and availability $[9,10]$. In our laboratory, several studies have been carried out [11] that showed and proved the feasibility of using glycerol as hole scavenger 
in the process. A recent study [12] also proposed some possible mechanisms of adsorption of glycerol on nanostructured titania, evidencing the importance of the defectivity of the oxide for its photoactivity and for its capacity to adsorb glycerol molecules. Glycerol resulted strongly adsorbable on the oxygen vacancies of $\mathrm{TiO}_{2}$ greatly influencing the behaviour of the structures during photocatalysis. The present work wants to give a contribution to the research in this field, widening the range of investigated wavelength of the incident radiation, in view of a possible optimization of the process and its addressing to practical applications. Actually, it must be considered that most of the investigations carried out so far in our laboratory, as well as most of the works present in the literature concerning nanostructured titania, were focused on the photo-activity of the samples in the UV range, where the $\mathrm{TiO}_{2}$ has its maximum performance.

For the purposes of possible practical applications that can exploit a larger percentage of the solar spectrum, it is useful to widen the field of investigation to different wavelengths of incident radiation. The present study investigates in particular a wavelength range from 340 to $400 \mathrm{~nm}$ to evaluate the effect of glycerol in this wider spectrum of conditions.

\section{Experimental}

2.1. Preparation of the Electrode. The electrodes were prepared starting from $\mathrm{Ti}$ foils $(0.25 \mathrm{~mm}$ thick, 99.7\% metal basis, Aldrich) and following the same procedure adopted and described elsewhere [13]. After a preliminary degreasing treatment, the Ti foil was electrochemically oxidised at $20 \mathrm{~V}$ in $0.4 \mathrm{M} \mathrm{NH}_{4} \mathrm{NO}_{3}+0.25 \mathrm{M} \mathrm{NaF}$ for three hours. Then, it was annealed $3 \mathrm{~h}$ at $400^{\circ} \mathrm{C}$, in air.

2.2. The Electrochemical Cell. A three-electrode cell was used for the electrochemical runs, in which the $\mathrm{TiO}_{2}$ sample was the working electrode; for all the experiments, its surface was shielded so that a nominal area of $1 \mathrm{~cm}^{2}$ was exposed to the electrolyte. A platinum grid and a saturated calomel electrode (SCE) constituted the counter and the reference electrodes, respectively; all the values of potential in the text are referred to SCE.

2.3. Experimental Runs. All the tests were performed in $\mathrm{KOH} 0.1 \mathrm{M}$ solution with or without the presence of glycerol 0.1 M. Electrochemical impedance spectroscopy (EIS) measurements were performed at different bias potentials in a frequency range from $100 \mathrm{kHz}$ to $0.1 \mathrm{~Hz}$. A frequency response analyzer (FRA, Model 7200 AMEL) coupled with a potentiostat (AMEL 7050) have been employed for these experiments. EIS data were quantitatively interpreted by the equivalent circuit approach. Zassist and ZSimpWin software packages were used to perform impedance experiments and data manipulation, respectively.

Polarisation curves were obtained in the dark or under irradiation by imposing a potential ramp from the open circuit voltage (OCV) to about $1 \mathrm{~V}$ with a scan rate of $5 \mathrm{mV} \mathrm{s}^{-1}$. Photocurrents were calculated by subtracting the values obtained in the dark from those measured under radiation.
The light source was a 300-W Xe lamp (Lot Oriel) the behaviour of the sample was studied using four filters with centre wavelength at $340,365,380$, and $400 \mathrm{~nm}$. The average light intensity striking on the surface of the electrode ranged from 1.5 to $11 \mathrm{mWcm}^{-2}$ depending on the filter. To compare results, photocurrents $(I)$ have been normalized with respect to the incident light power $(P)$.

Voltammetric scans were performed starting from the OCV towards the negative potential down to $-1.5 \mathrm{~V}$, with a scan rate of $200 \mathrm{mV} \mathrm{s}^{-1}$.

The effect of the adsorption of glycerol was particularly investigated by performing different scans after that sample was maintained in contact with $0.1 \mathrm{M}$ glycerol solution for increasing intervals of time. The effect of desorption was investigated maintaining the sample in $\mathrm{KOH} 0.1 \mathrm{M}$ solution.

For all the runs, chemicals were of reagent grade (supplied by Aldrich) and they were used as received.

\section{Results and Discussion}

In order to use glycerol as a sacrificial agent in the process of hydrogen production by solar energy, a deep understanding on the working mechanism of the electrodes and on the interactions between glycerol and nanotubular $\mathrm{TiO}_{2}$ has to be gained. For some years, our work has been aimed to this end; we found out that photo-catalytic response of $\mathrm{TiO}_{2}$ nanotubular arrays depends on a wide number of factors related to the specific conditions of synthesis and to the morphological and electronic properties of the samples [11-14]. Moreover, we also investigated the opportunity to enhance the photo-electrochemical yield through the use of glycerol as hole scavenger $[11,12]$. Depending on the electrodes, different percentage increases in the current yield were obtained: in some cases the addition of glycerol showed to be of scarce effect [11]; in other cases, we obtained increases of more than the $100 \%$ [12]. In the present work the study has been focused to the behaviour of those samples that gave the best performance, by investigating in detail the responses in photocurrent tests in an extended range of wavelength. The results from these experiments, along with those derived from voltammetric and EIS analysis are presented in the following.

Figure 1 reports the results of the photo-electrochemical tests performed at different wavelengths, in solutions without and with the presence of glycerol as hole scavenger. Results obtained at $365 \mathrm{~nm}$ are in agreement with those obtained for a sample synthesized under the same operative conditions [12]. In all the cases, curves always show an initial increasing trend of photocurrent with the applied potential and a second part less dependent on it. Moreover, potential value being the same, photocurrents decrease with the wavelength according to the absorption coefficient of the $\mathrm{TiO}_{2}$.

To evaluate quantitatively the enhancement of photocurrent after the addition of glycerol, the ratio $\mathbf{j}$ between the photocurrent responses in presence $\left(I_{G}\right)$ and in absence of glycerol $\left(I_{\mathrm{KOH}}\right)$ has been compared at two significant potential values: the first $\left(E_{1}\right)$ where photocurrents are highly potential dependent was fixed as $0.05 \mathrm{~V}$ higher than the current onset, and the second $\left(E_{2}\right)$ in the range where 


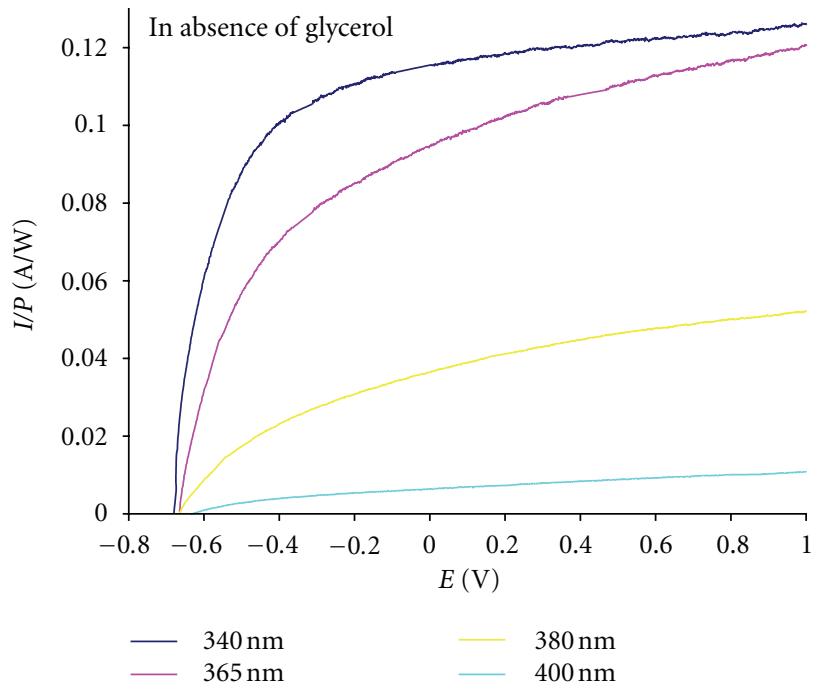

(a)

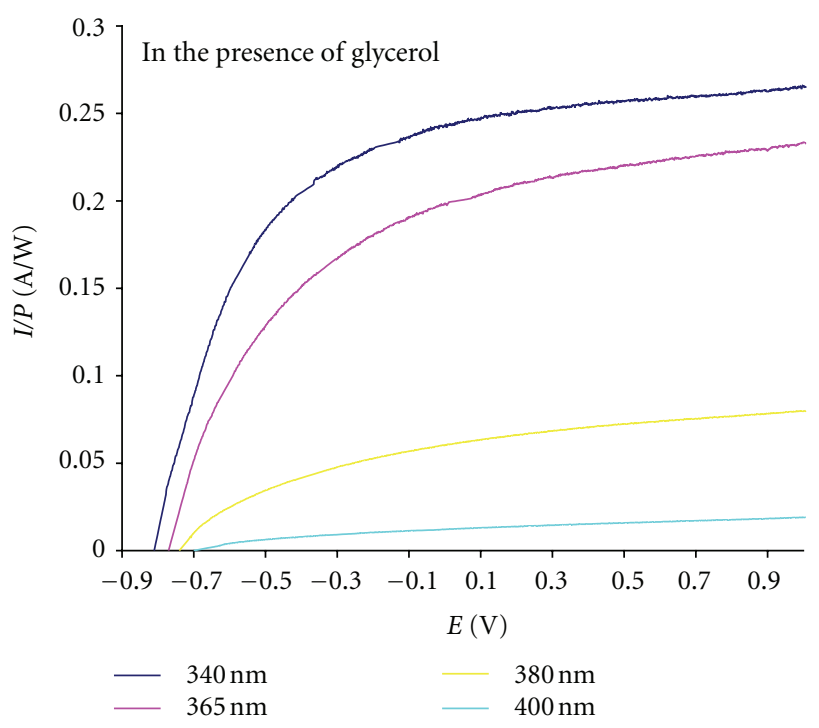

(b)

FIGURE 1: Trend of photocurrents (normalized to incident power light) versus potential, at different wavelengths, in absence and in presence of glycerol.

currents have reached their steady state values was fixed at $1 \mathrm{~V}$ from the current onset. Data are reported in Table 1. As it can be seen, a positive effect of glycerol, indicated by the j values greater than unit, is achieved at all the wavelengths: higher $\mathbf{j}$ values are measured, in the range of $2-4$, near the on set potential, confirming the positive effect of glycerol in reducing the high rate of recombination of photogenerated charges occurring at this potential. The effect of glycerol is less evident in the presence of a higher external applied potential: values of $\mathbf{j}$ go down around the value of 2 so highlighting how the applied potential also contributed to the lowering of the charge recombination process.

If the trend of $\mathbf{j}$ with the wavelength is considered, it is possible to note that while at the higher potential $\left(E_{2}\right)$, the ratio $\mathbf{j}$ remains more or less stable around 2 at all the considered wavelengths, and at the lower potential $\left(E_{1}\right)$, the best performances are obtained in the range of visible light. This is a very remarkable result especially if we consider how the applied potential affects the energetic yield of an electrically driven photoprocess [15]:

$$
\varepsilon=\frac{I\left(E_{\mathrm{rev}}{ }^{0}-\left|E_{\mathrm{app}}\right|\right)}{P},
$$

in which $E_{\mathrm{rev}}{ }^{0}=1.23 \mathrm{~V}$ is the standard reversible potential for water splitting, and $P$ is the light power.

As it can be observed, the yield takes into account the net power output obtained in the process, being $E_{\text {appl }}$ the difference between the bias potential and the OCV under the irradiated power light. From this equation it is evident that the possibility to work at lower potential has to be preferred in this kind of processes.

In the present case, the results obtained indicate that the presence of glycerol particularly favours conditions of lower applied potential and higher wavelength that are the best conditions to carry out the considered process in an ecofriendly way.

To better understand the working mechanism of the electrode and the interactions occurring between glycerol and nanotubular $\mathrm{TiO}_{2}$, EIS measurements were carried out at the irradiated sample; also in this case, two different ranges of potential were investigated, near to the OCV and at higher values where the saturation current was achieved.

Figure 2 reports the spectra, in terms of Bode and Nyquist plots, obtained in absence and in presence of glycerol, at the potential of $0.5 \mathrm{~V}$.

No significant differences are evident in Bode phase angle diagrams. As the Bode modulus versus frequency plot is considered, at the higher frequencies, where as it is known the solution resistance $\left(R_{S}\right)$ dominates the impedance, values overlapped, confirming the almost equal resistance of the solution, whose conductivity is given mainly by the supporting electrolyte. As the frequency decreases, the curves still remain overlapped; only at values lower than about $1 \mathrm{~Hz}$, a slight separation of the modulus appeared, whose meaning can be better seen on the Nyquist plot, where, at the lower frequencies, the semicircle related to charge transfer occurring at the interface electrode-electrolyte appears. According to the higher photocurrents obtained in presence of glycerol, the semicircle related to the reaction happening at the electrode-electrolyte interfaces shows a smaller diameter when glycerol is present, indicating a more easy charge transfer with the solution.

Other important information can be gathered from the EIS performed at the potential near the OCV, (Figure 3); under this potential the extent of the occurring reactions is very low, and more interesting information can be obtained on the capacitive behaviour of the sample.

Referring to Bode diagram of modulus, also at this potential, the values overlapped at the higher frequencies, but 
TABLE 1: $I_{G} / I_{\mathrm{KOH}}$ values obtained at the different wavelengths and at two different potentials.

\begin{tabular}{llccccc}
\hline \multirow{2}{*}{$j=I_{G} / I_{\mathrm{KOH}}$} & & 340 & 365 & Wavelength $(\mathrm{nm})$ & 380 & 400 \\
\hline \multirow{2}{*}{ Potential $(\mathrm{V})$} & $E_{1}$ & 2.4 & 3.1 & 3 & 4 \\
& $E_{2}$ & 2.1 & 2.1 & 1.8 & 1.9 \\
\hline
\end{tabular}

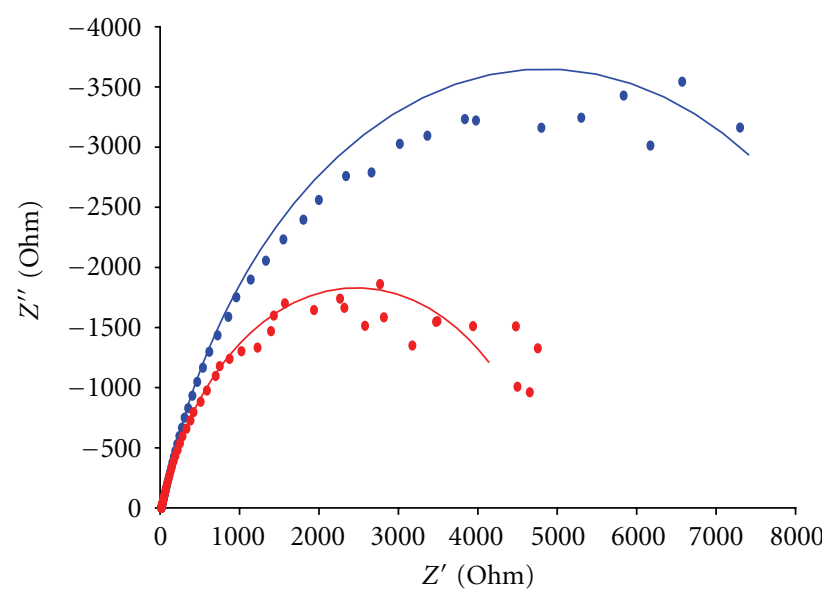

(a)

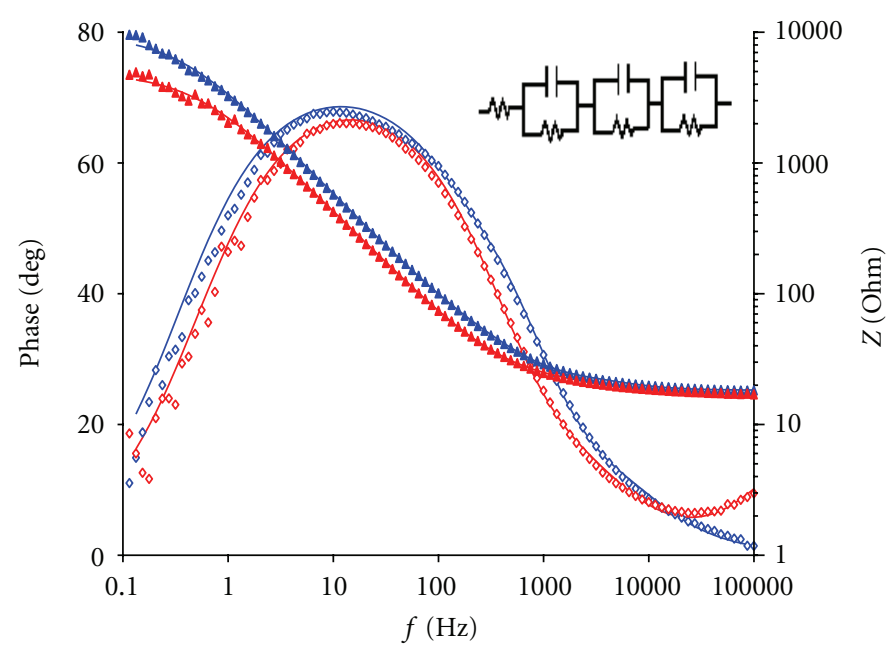

(b)

FIGURE 2: EIS results under irradiation: (a) Nyquist plots; (b) phase angle (empty symbols) and modulus (solid symbols) Bode plots; $E=$ $0.5 \mathrm{~V}, \lambda=365 \mathrm{~nm}$. Blue symbols: in absence of glycerol. Red symbols: in presence of glycerol. Solid lines refer to the model fit.

in this case, curves are differentiated starting from about $80 \mathrm{~Hz}$, being values obtained in presence of glycerol lower. In particular, a wide range of the curves with linear trend can be identified with negative slope equal to unit; according to the theory, an indication of the value of the double layer capacitance $\left(1 / C_{\mathrm{dl}}\right)$ can be obtained from the intersection of the straight line with the $y$-axis. As expected, the increased $C_{\mathrm{dl}}$ in presence of glycerol suggests a significant adsorption of glycerol at the electrode surface.

The presence of adsorbed glycerol can be confirmed also by the analysis of Nyquist plot at the intermediated frequencies, as visible in Figure 3, where a suitable magnification of the plot has been presented. In this range of frequencies, a linear trend of the data is detected, whose unit slop indicated that diffusion processes of the reactive species inside the tubes may be relevant, both in absence and in presence of glycerol.

Data were quantitatively interpreted by means of the equivalent circuit approach in which the behaviour of the experimental system was compared with the one of a suitable electrical circuit (equivalent circuit) submitted to the same input potential signal. A fitting procedure between the response of the experimental system and that of the equivalent circuit allowed to evaluate the circuital elements to which a physical meaning can be attributed.

In the present case, an equivalent circuit with threetime constants was able to interpret the data obtained under irradiation in the two ranges of potentials, respectively, at OCV $\left(R_{S}\left(R_{1} C_{1}\right)\left(R_{2} C_{2}\right) W\right)$ and under saturation current regime $\left(R_{S}\left(R_{1} C_{1}\right)\left(R_{2} C_{2}\right)\left(R_{3} C_{3}\right)\right)$.
It must be observed that both the circuits include the resistance $R_{S}$ which takes into account the solution resistance which was found in all cases very low due to the high conductivity of the solution. The other elements are used to represent the response of the electrode film and the electrode/solution interphase. Tables 2 and 3 resume the values of the circuital parameters calculated by the fit of experimental data.

For the circuits interpreting the system at OCV, the presence of an element with high time constant $\left(R_{2} C_{2}\right)$, along with the Warburg element may describe a slow process of diffusion and adsorption. Actually, the presence of the W element highlights the relevance of diffusion effects; the following relationship can be written for the impedance of the $\mathrm{W}$ element:

$$
Z_{W}=\frac{1}{\sqrt{2 \omega} Y_{0}}-j \frac{1}{\sqrt{2 \omega} Y_{0}},
$$

in which the Warburg coefficient $Y_{0}$ depends on typical properties of the system, such as diffusivity and concentration of the active specie, electrode area involved, and temperature.

In order to describe data at higher potential, in addition to the $R_{f} C_{f}$, other two elements are needed. In this case, a constant phase element (CPE) was needed to represent the nonideal behavior of the capacitor. Actually, impedance of a $\mathrm{CPE}$ may be expressed as

$$
\left(Z_{\mathrm{CPE}}=\frac{1}{Q(j \omega)^{n}}\right)
$$




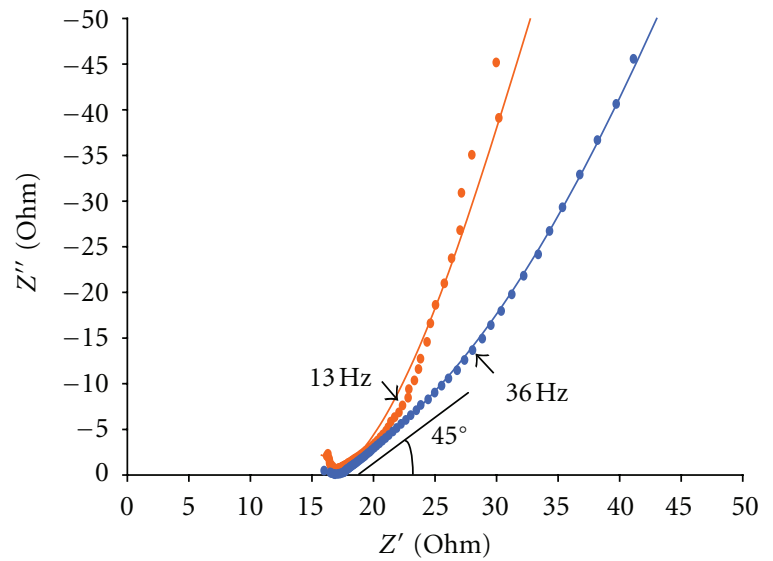

(a)

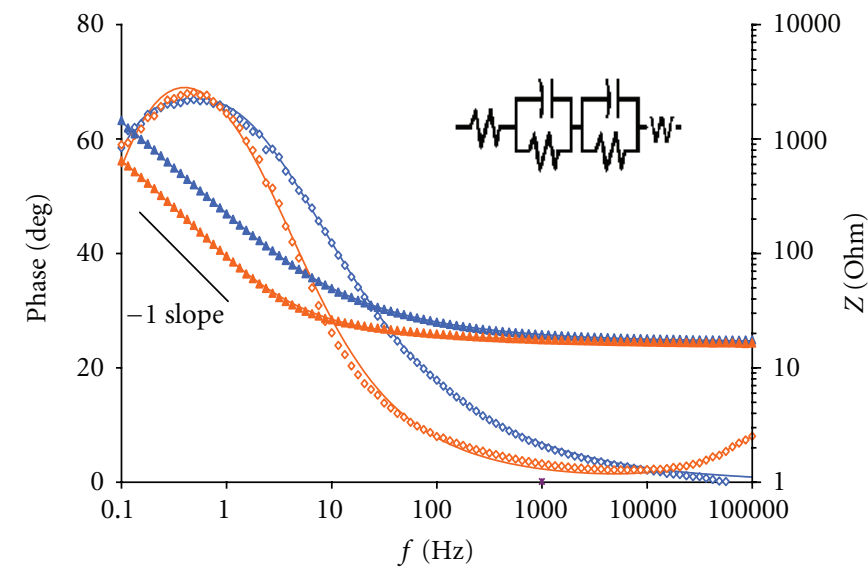

(b)

FIGURE 3: EIS results under irradiation: (a) Nyquist plots; (b) phase angle (empty symbols) and modulus (solid symbols) Bode plots; $E=$ OCV,$\lambda=365 \mathrm{~nm}$. Blu symbols: in absence of glycerol. Orange symbols: in presence of glycerol. Solid lines refer to the model fit.

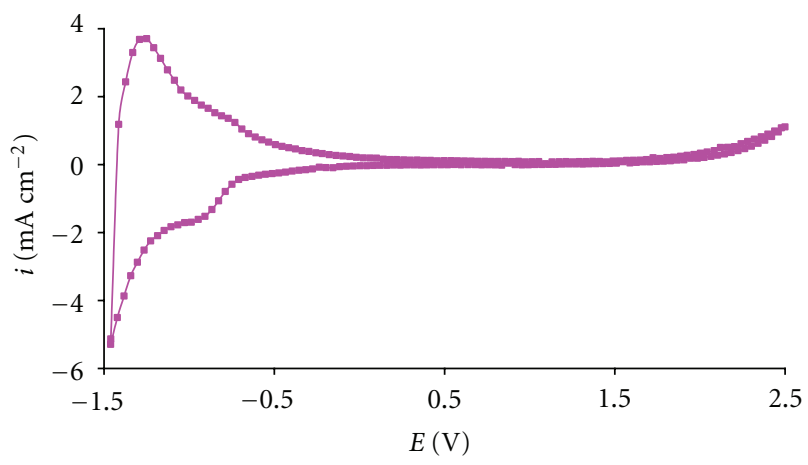

Figure 4: Example of cyclic voltammetry carried out at $0.1 \mathrm{M}$ glycerol solution at $\mathrm{TiO}_{2}$ electrode.

being $n=1$ for a perfect capacitor. Its time constant $\left(\tau_{3}=(\mathrm{RQ})^{1 / n}\right)$ in the order of seconds, along with that of the $R_{2} C_{2}$ ( $\tau_{2}$ in the order of $\mu \mathrm{s}$ ) indicate fast processes occurring at the electrode at this potential value. Accordingly, a lower diameter is recorded in the loops of the Nyquist plots compared to that recorded at the OCV and higher currents are measured.

To deeply investigate adsorption process, voltammetric scans were carried out in $\mathrm{KOH}$ solutions containing glycerol in the range of negative potentials from the OCV to about $-1.5 \mathrm{~V}$. An example of these results is shown in Figure 4. Previous studies [12] showed that the height of the cathodic peak observed at about $-1 \mathrm{~V}$ may indicate the extent of the adsorption of glycerol on the surface of $\mathrm{TiO}_{2}$. In this case, $\mathrm{CV}$ runs were performed after the sample was contacted for increasing times by glycerol solution. CV runs were then repeated after desorption in $\mathrm{KOH}$ solvent.

In Table 4, the height of the cathodic peak is reported as a function of the adsorption and desorption times. Data suggest a slow adsorption process occurring on the superficial state of $\mathrm{TiO}_{2}$ nanotubes, and a quite reversible, even slower desorption process.
Also in this case, a series of EIS measurements have been carried out, with and without glycerol in the dark, in order to study the behaviour of the sample as a function of the adsorption time, and the results interpreted by suitable equivalent circuits.

Figure 5 shows the Nyquist plots obtained at the sample maintained in contact with glycerol alkaline solution for increasing intervals of time. In the same figure, Nyquist plot obtained in a $\mathrm{KOH}$ solution is reported as comparison. Also in this case, a suitable magnification of the diagram has been chosen to highlight the range of major changes caused by the absorption process.

As well visible in Figure 5, only after $20 \mathrm{~h}$, glycerol adsorption noticeably modifies the first semicircle of the plot. At the higher frequencies (data not reported), the trend of impedance values is not particularly meaningful showing, as expected, a simply resistive behaviour, since reactions do not occur in the system without illumination and near the open circuit potential.

As shown in Figure 5(b), the data obtained after 20 and 40 hours of desorption tend to merge with those obtained before the glycerol adsorption; the adsorption process is reversible but the desorption process results to be quite slow.

A quantitative analysis of the kinetics of adsorption is reported in Table 5, where the circuital parameters are compared. In particular, as already outlined in the previous figures, the sample resistance $R_{f}$ increases with the adsorption of glycerol and then it decreases after desorption. The fact that the adsorption of glycerol leads to the increase in the material resistance supports the hypothesis [12], that it is preferentially adsorbed on the superficial states responsible of the conductibility of the electrode.

Also in this case, the high value of the time constant $\tau 2$ as well as the presence of a Warburg element indicate that, in these conditions, the adsorption represents the rate determining step of the whole process. However, as the specific values of $Y_{0}$ are compared, their variation is quite difficult to be interpreted; in fact, as already pointed out, although the coefficient $Y_{0}$ could be related to the diffusion coefficient of 
TABLE 2: Circuit parameters obtained by fitting the experimental impedance results with the selected equivalent circuit. Data obtained under irradiation $(\lambda=365 \mathrm{~nm})$ in the presence and in absence of glycerol, $E=\mathrm{OCV}$.

\begin{tabular}{lcccccc}
\hline & & \multicolumn{2}{c}{$E=\mathrm{OCV}$} \\
& $C_{f}\left(\mu \mathrm{F} \mathrm{cm}^{-2}\right)$ & $R_{f}\left(\Omega \mathrm{cm}^{2}\right)$ & $C_{2}\left(\mu \mathrm{F} \mathrm{cm}^{-2}\right)$ & $R_{2}\left(\Omega \mathrm{cm}^{2}\right)$ & $\tau_{2}(\mathrm{~s})$ & $Y_{0}\left(\mu \mathrm{S} \mathrm{s} \mathrm{cm}^{-2}\right)$ \\
\hline $\mathrm{KOH}$ & 10 & 17.2 & 1200 & 2544 & 3.04 & 4700 \\
$\mathrm{G}$ & 0.23 & 16.66 & 2460 & 1041 & 2.55 & 14500 \\
\hline
\end{tabular}

TABLE 3: Circuit parameters obtained by fitting the experimental impedance results with the selected equivalent circuit. Data obtained under irradiation $(\lambda=365 \mathrm{~nm})$ in the presence and in absence of glycerol, $E=0.5 \mathrm{~V}$.

\begin{tabular}{lccccccccc}
\hline & \multicolumn{1}{c}{$E=0.5 \mathrm{~V}$} \\
& $C_{f}\left(\mu \mathrm{F} \mathrm{cm}^{-2}\right)$ & $R_{f}\left(\Omega \mathrm{cm}^{2}\right)$ & $C_{2}\left(\mu \mathrm{F} \mathrm{cm}^{-2}\right)$ & $R_{2}\left(\Omega \mathrm{cm}^{2}\right)$ & $\tau_{2}(\mathrm{~s})$ & $Q_{3}\left(\mu \mathrm{S} \mathrm{s}^{n} \mathrm{~cm}^{-2}\right)$ & $n_{3}$ & $R_{3}\left(\Omega \mathrm{cm}^{2}\right)$ & $\tau_{3}(\mathrm{~s})$ \\
\hline $\mathrm{KOH}$ & 82.5 & 24.93 & 10.1 & 2.40 & 24 & 55.8 & 0.83 & 9619 \\
$\mathrm{G}$ & 0.13 & 17.63 & 22.1 & 2.45 & 54 & 78.8 & 0.47 \\
\hline
\end{tabular}

TABLE 4: Heights of the cathodic peak observed at $-1 \mathrm{~V}$ as a function of the adsorption $\left(t_{\mathrm{ads}}\right)$ and desorption $\left(t_{\mathrm{des}}\right)$ times.

\begin{tabular}{lcccccc}
\hline & KOH 0.1 M & \multicolumn{3}{c}{ KOH 0.1 M + glycerol 0.1 M } & \multicolumn{3}{c}{ KOH 0.1 M } \\
& & $t_{\text {ads }}=0$ & $t_{\text {ads }}=4 \mathrm{~h}$ & $t_{\text {ads }}=20 \mathrm{~h}$ & $t_{\text {des }}=20 \mathrm{~h}$ & $t_{\text {des }}=40 \mathrm{~h}$ \\
\hline Height of the cathodic peak (V) & -1.8 & -3 & -3.5 & -4 & -3 & -2 \\
\hline
\end{tabular}

TABLE 5: Circuit parameters obtained by fitting the experimental impedance results with the selected equivalent circuit. Data obtained in the dark at the OCV potential, as a function of the adsorption $\left(t_{\mathrm{ads}}\right)$ and desorption $\left(t_{\mathrm{des}}\right)$ times.

\begin{tabular}{|c|c|c|c|c|c|c|}
\hline & $C_{f}\left(\mu \mathrm{F} \mathrm{cm}^{-2}\right)$ & $R_{f}\left(\Omega \mathrm{cm}^{2}\right)$ & $C_{2}\left(\mu \mathrm{F} \mathrm{cm}^{-2}\right)$ & $R_{2}\left(\Omega \mathrm{cm}^{2}\right)$ & $\tau_{2}(\mathrm{~s})$ & $Y_{0}\left(\mathrm{mS} \mathrm{s}^{0.5} \mathrm{~cm}^{-2}\right)$ \\
\hline $\mathrm{KOH}$ & 0.031 & 11.83 & 940 & 6939 & 6.5 & 8.8 \\
\hline$t_{\mathrm{ads}}=0$ & 0.026 & 12.15 & 939 & 7349 & 6.90 & 6.5 \\
\hline$t_{\mathrm{ads}}=4 \mathrm{~h}$ & 0.19 & 12.5 & 1200 & 10600 & 12.74 & 14.6 \\
\hline$t_{\mathrm{ads}}=20 \mathrm{~h}$ & 0.021 & 16.11 & 900 & 4956 & 4.46 & 4.95 \\
\hline$t_{\mathrm{des}}=40 \mathrm{~h}$ & 0.02 & 12.36 & 206 & 13100 & 2.70 & 3.01 \\
\hline
\end{tabular}

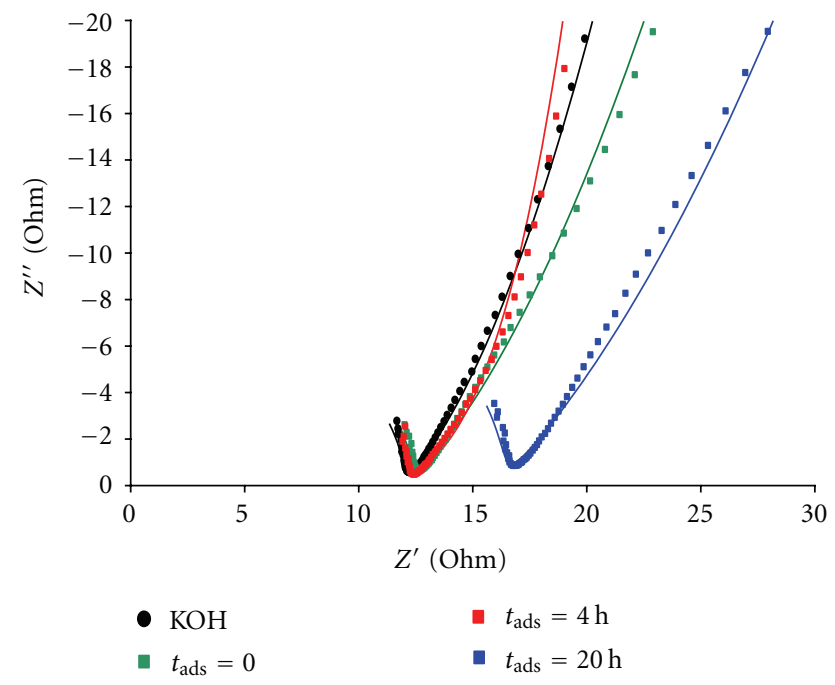

(a)

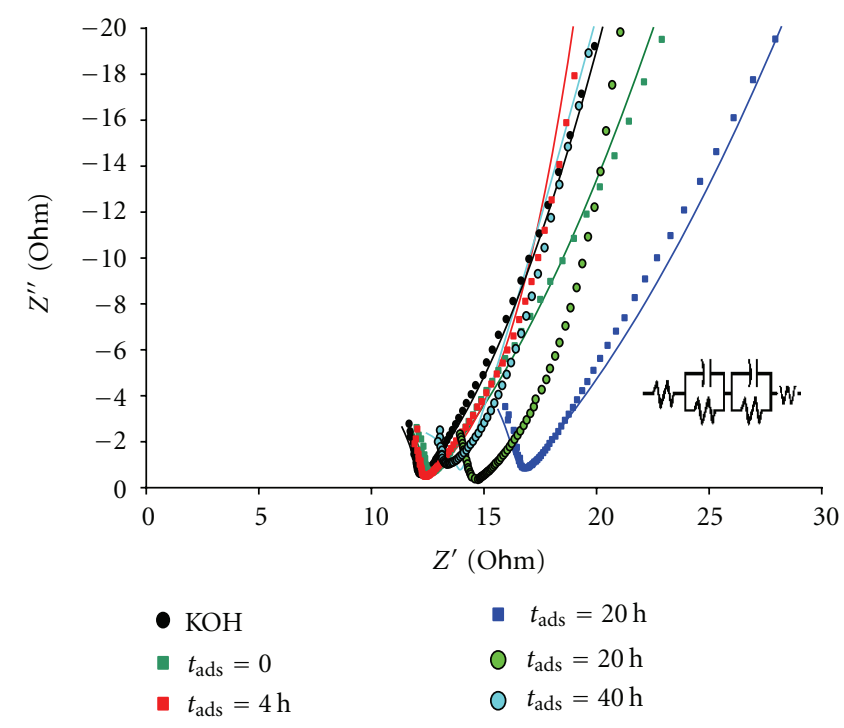

(b)

FIGURE 5: (a) Effect of G adsorption on the Nyquist plots; each curve is obtained after the sample was maintained in contact with (G) = 0.1 M alkaline solution for increasing intervals of time $t$. Black symbols refer to test performed in the solvent without G. (b) Effect of G desorption. Solid lines refer to the model fit. 
the species, other factors are included in its formula, such as the area or concentration of the active species, which, in a complex structure like this, are not easy to be identified.

\section{Conclusions}

This work deepened the study carried out in our laboratory on photo catalytic hydrogen production enhanced by the presence of glycerol as sacrificial agent. Results have been presented in order to better understand the working mechanism of the electrodes and the interactions occurring between glycerol and nanotubular $\mathrm{TiO}_{2}$. Results obtained highlighted that adsorption process has to be considered and correlated to the morphology and the defectivity of the sample. On the one hand greater defectivity increases the adsorption capacity of the electrode, which is necessary to ensure increased efficiencies of photocurrent, but on the other hand an excessive reduction in conductivity must be prevented in order not to affect the sample performance. Further studies are still on the way on different electrodes in order to optimise morphology and defectivity of the sample to be destined to the specific process.

\section{References}

[1] M. Pagliaro, R. Ciriminna, H. Kimura, M. Rossi, and C. Della Pina, "From glycerol to value-added products," Angewandte Chemie International, vol. 46, no. 24, pp. 4434-4440, 2007.

[2] M. G. Alvarez, A. M. Segarra, S. Contreras, J. E. Sueiras, F. Medina, and F. Figueras, "Enhanced use of renewable resources: transesterification of glycerol catalyzed by hydrotalcite-like compounds," Chemical Engineering Journal, vol. 161, no. 3, pp. 340-345, 2010.

[3] R. Rudie, "Corgill Dow sows seeds of future fibers; will build \$300 million PLA polymer plant," International Fiber Journal, vol. 15 , no. 1 , pp. 8-12, 2000.

[4] M. A. Dasari, P. P. Kiatsimkul, W. R. Sutterlin, and G. J. Suppes, "Low-pressure hydrogenolysis of glycerol to propylene glycol," Applied Catalysis A, vol. 281, no. 1-2, pp. 225-231, 2005.

[5] V. Augugliaro, H. A. H. El Nazer, V. Loddo et al., "Partial photocatalytic oxidation of glycerol in $\mathrm{TiO} 2$ water suspensions," Catalysis Today, vol. 151, no. 1-2, pp. 21-28, 2010.

[6] H. Wu and Z. Zhang, "Photoelectrochemical water splitting and simultaneous photoelectrocatalytic degradation of organic pollutant on highly smooth and ordered $\mathrm{TiO}_{2}$ nanotube arrays," Journal of Solid State Chemistry, vol. 184, no. 12, pp. 3202-3207, 2011.

[7] M. Canterino, I. Di Somma, R. Marotta, R. Andreozzi, and V. Caprio, "Energy recovery in wastewater decontamination: simultaneous photocatalytic oxidation of an organic substrate and electricity generation," Water Research, vol. 43, no. 10, pp. 2710-2716, 2009.

[8] A. Galińska and J. Walendziewski, "Photocatalytic water splitting over $\mathrm{Pt}-\mathrm{TiO}_{2}$ in the presence of sacrificial reagents," Energy and Fuels, vol. 19, no. 3, pp. 1143-1147, 2005.

[9] S. Rani, S. C. Roy, M. Paulose et al., "Synthesis and applications of electrochemically self-assembled titania nanotube arrays," Physical Chemistry Chemical Physics, vol. 12, no. 12, pp. 2780-2800, 2010.

[10] P. Roy, S. Berger, and P. Schmuki, " $\mathrm{TiO}_{2}$ nanotubes: synthesis and applications," Angewandte Chemie International, vol. 50, no. 13 , pp. 2904-2939, 2011.
[11] S. Palmas, A. M. Polcaro, J. R. Ruiz, A. Da Pozzo, M. Mascia, and A. Vacca, " $\mathrm{TiO}_{2}$ photoanodes for electrically enhanced water splitting," International Journal of Hydrogen Energy, vol. 35, no. 13, pp. 6561-6570, 2010.

[12] S. Palmas, A. Da Pozzo, M. Mascia, A. Vacca, P. C. Ricci, and R. Matarrese, "On the redox behaviour of glycerol at $\mathrm{TiO}_{2}$ electrodes," Journal of Solid State Electrochemistry, vol. 16, pp. 2493-2502, 2012.

[13] S. Palmas, A. Da Pozzo, M. Mascia et al., "Effect of the preparation conditions on the performance of $\mathrm{TiO}_{2}$ nanotube arrays obtained by electrochemical oxidation," International Journal of Hydrogen Energy, vol. 36, no. 15, pp. 8894-8901, 2011.

[14] S. Palmas, A. Da Pozzo, F. Delogu, M. Mascia, A. Vacca, and G. Guisbiers, "Characterization of $\mathrm{TiO}_{2}$ nanotubes obtained by electrochemical anodization in organic electrolytes," Journal of Power Sources, vol. 204, pp. 265-272, 2012.

[15] O. K. Varghese and C. A. Grimes, "Appropriate strategies for determining the photoconversion efficiency of water photoelectrolysis cells: a review with examples using titania nanotube array photoanodes," Solar Energy Materials and Solar Cells, vol. 92, no. 4, pp. 374-384, 2008. 


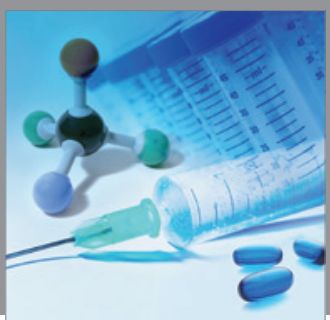

International Journal of

Medicinal Chemistry

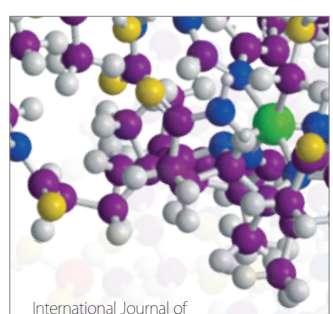

Carbohydrate Chemistry

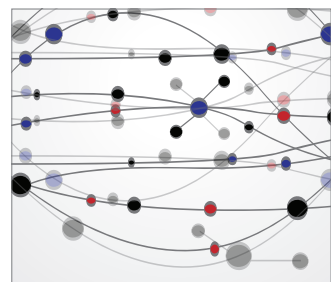

The Scientific World Journal
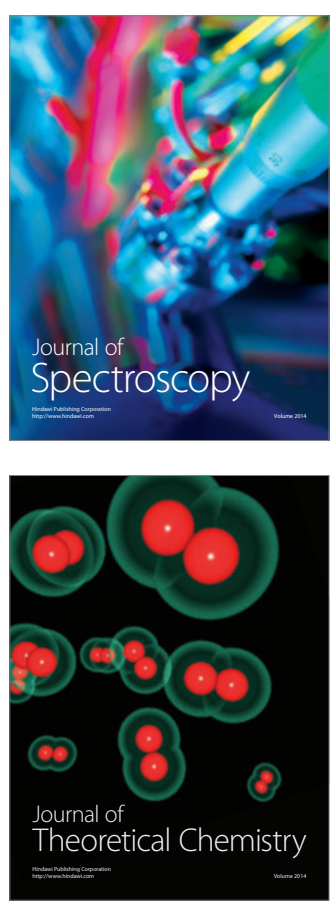
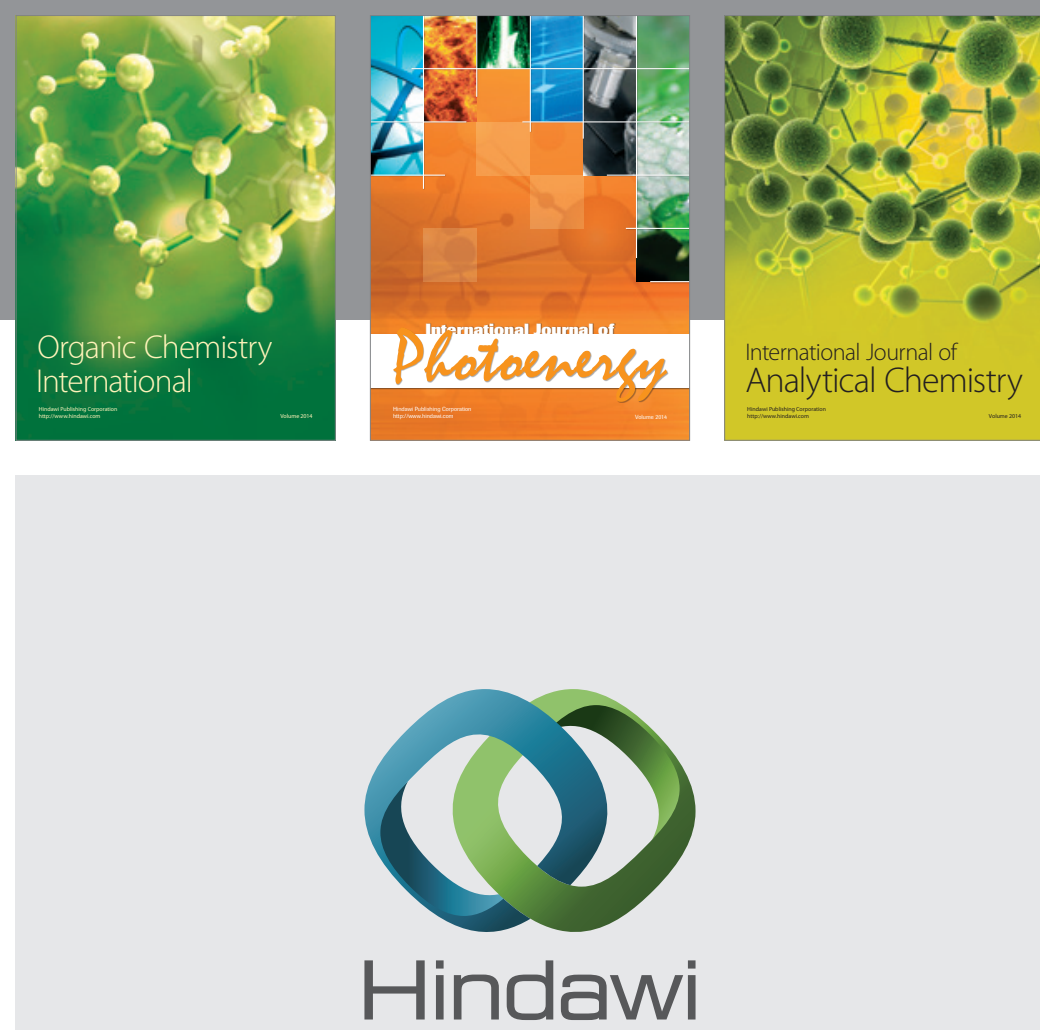

Submit your manuscripts at

http://www.hindawi.com
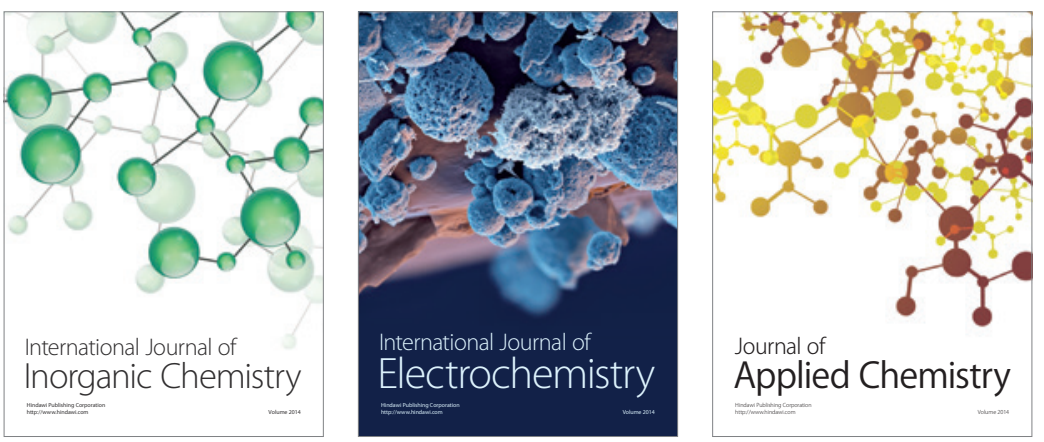

Journal of

Applied Chemistry
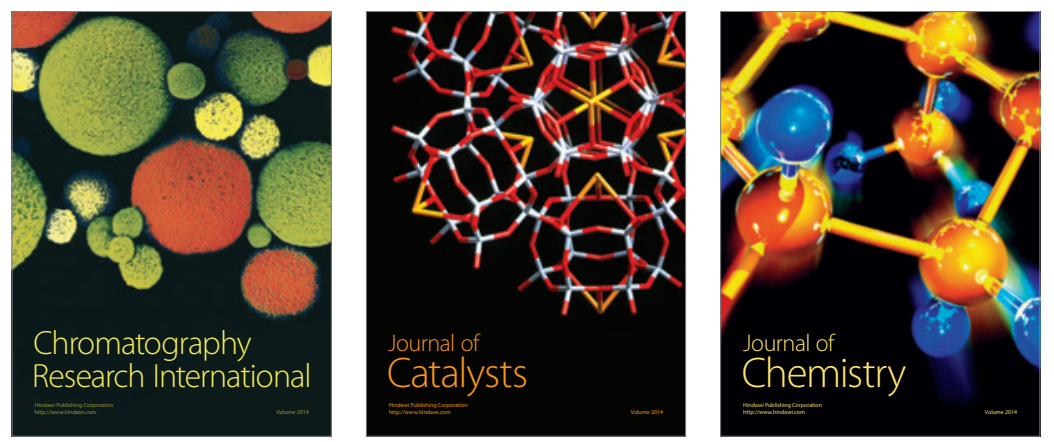
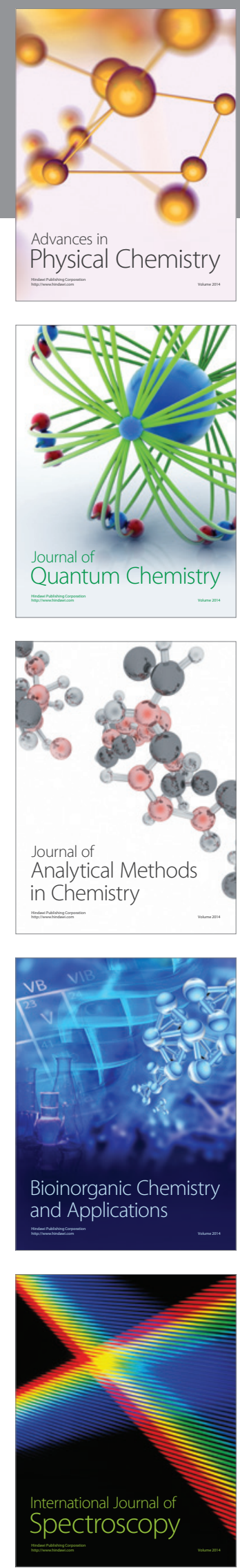